\title{
Nucleon pairing in Sn isotopes
}

\author{
L. Imasheva ${ }^{1}$, B. Ishkhanov ${ }^{1,2}$, M. Stepanov ${ }^{1}$, and T. Tretyakova ${ }^{2, a}$ \\ ${ }^{1}$ Faculty of Physics, Lomonosov Moscow State University, Moscow, 119991 Russia \\ ${ }^{2}$ Skobeltsyn Institute of Nuclear Physics, Lomonosov Moscow State University, Moscow, 119991 Russia
}

\begin{abstract}
The systematics of excited states in Sn isotopes are discussed on basis of pairing interaction in nuclei. Nucleon paring leads to formation of excited states multiplets. The estimation of multiplet splitting based on experimental nuclear masses allows one to calculate the position of excited states with different seniority in $\delta$-approximation. The wide systematics of the spectra of Sn isotopes gives a possibility to check the pairing interaction for different subshells and consider the multiplets of excited states in the neutron-rich isotopes far from stability.
\end{abstract}

\section{Introduction}

The chain of tin isotopes is the longest one at the nuclear map. Having the magic proton number $Z=50$, this element has ten stable isotopes and its chain stretches from ${ }^{99} \mathrm{Sn}$ up to ${ }^{138} \mathrm{Sn}$ (49 $\geq N \geq 88$ ). The magic nuclei with a certain degree of simplification can be explored as a double-closed shell nuclei plus or minus several nucleons. In this approach the low-energy structure should reflect the properties of the pairing interaction very clear. Indeed, the even-even $\mathrm{Sn}$ isotopes are characterised by the significant and nearly constant energy gap of about $1.2 \mathrm{MeV}$, which separates the ground state $J^{\pi}=0^{+}$and the first excited state. Moreover, the state $2_{1}^{+}$is single in the region up to $2 \mathrm{MeV}$ and it can be interpreted as a broken pair of neutrons recoupled to $J^{\pi}=2^{+}$[1]. On the other hand, the presence of group of levels with $J^{\pi}=0^{+}, 2^{+}$and $4^{+}$ near $2 \mathrm{MeV}$ allows one to interpret the state $2_{1}^{+}$in even isotopes ${ }^{110-124} \mathrm{Sn}$ as a one-phonon quadrupole vibrational state. The development of various approaches with taking into account one- and two-phonon degrees of freedom has achieved considerable results in the description of the state $2_{1}^{+}$properties in Sn isotopes [2,3]. Such collective interpretation simply implies a strongly correlated structure for the recoupled $J^{\pi}=2^{+}$pair $[1,4]$.

Another feature of the chain of tin isotopes is the fact that the double-closed shell $\mathrm{Sn}$ isotopes with the neutron magic numbers $N=50$ and 82 are unstable. The lifetime of the proton-rich isotope ${ }^{100} \mathrm{Sn}$ is about a second and there is no any information on its excitations. The neutron-rich isotope ${ }^{132} \mathrm{Sn}$ has the half-life time $T_{1 / 2}=39.7 \pm 0.8 \mathrm{~s}$, so the experimental information is more extensive. The neutron shell $50 \geq N \geq 82$ in tin isotopes has a complicated structure. The systematic features of low-lying levels in odd isotopes shows the filling of single-particle shell model states. The most of stable tin isotopes cor-

\footnotetext{
a e-mail: tretyakova@sinp.msu.ru
}

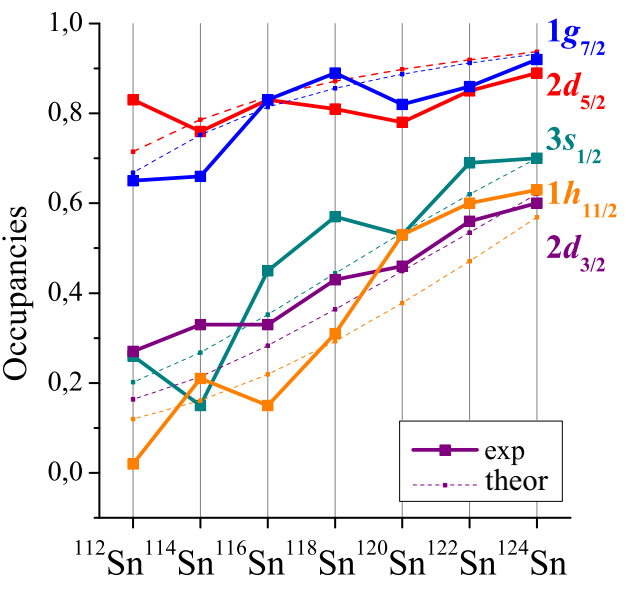

Figure 1. (Color online) Experimental and theoretical values of the single-particle occupation numbers [5].

respond to closed $1 g_{7 / 2}$ and $2 d_{5 / 2}$ subshells and present some mixed states of $3 s_{1 / 2}, 2 d_{3 / 2}$ and $1 h_{11 / 2}$. The filling of this states cannot be considered consistent and evidenced by the dynamics of the populations of the states. The appropriate experimental data on the occupancies $v^{2}$ of the neutron subshells in the even $\mathrm{Sn}$ isotopes are shown in Fig. 1 together with the results of calculations [5]. As one can see from Fig. 1 the better agreement of the $v^{2}$ values for the odd $\mathrm{Sn}$ nuclei close to ${ }^{100} \mathrm{Sn}$ is observed for those theoretical single-particle spectra, where the neutron $2 d_{5 / 2}$ subshell is lower than the $1 g_{7 / 2}$ one. It should be noted that despite the fact that the theoretical $A$-dependences of $v^{2}$ coefficients are smoother as compared with the experimental data, they reproduce the major trend - the simultaneous filling of $3 s_{1 / 2}, 2 d_{3 / 2}$ and $1 h_{11 / 2}$ subshells. 


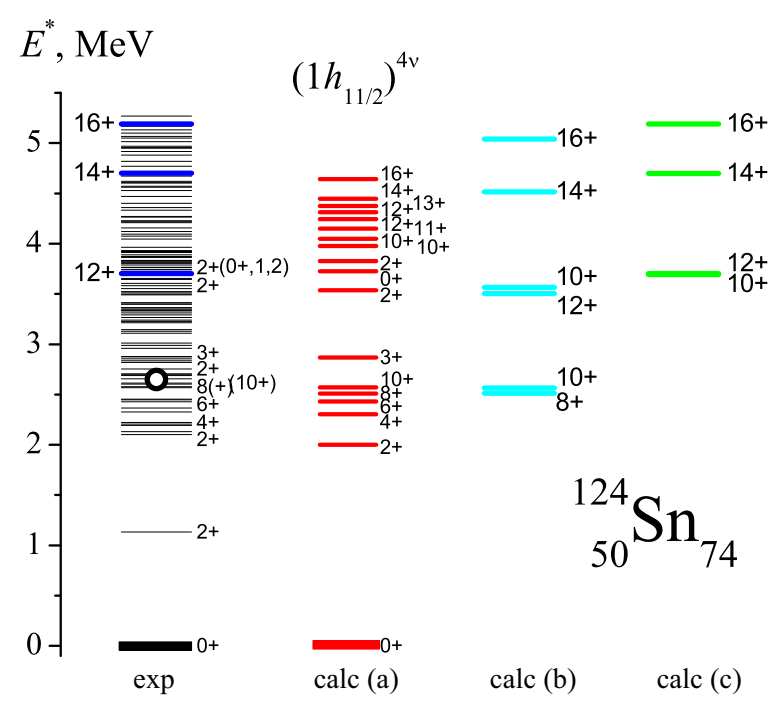

Figure 2. (Color online) Experimental spectrum of ${ }^{124} \mathrm{Sn}[6,7]$ (left column). Blue lines denote results from [8]. The open circle shows the pairing energy $\Delta_{n n}$ estimated from data in Ref. [9]. Calculations: (a) this work (see text), (b) from [8], (c) from [10].

The separation energy of the neutron pair $S_{n n}$ exhibits a smooth dynamics with $N$ too and indicates that the substructure of shell is smoothed away by pairing correlations [11]. These shell structure features and the presence of various collective degrees of freedom leads to a very complex spectra above $2 \mathrm{MeV}$. But nevertheless, a neutron pairing in $1 h_{11 / 2}$ state should lead to specific high- $J$ levels in the even ${ }^{120-130} \mathrm{Sn}$ isotopes.

\section{Nuclear pairing and seniority}

The pair of identical nucleons in a subshell with angular momentum $j$ over a core of double-closed shells forms the set of levels, named ground state multiplet, with even values of the total spin $J$

$$
J=0,2,4, \ldots J_{\max }, J_{\max }=2 j-1 .
$$

In the case of non-interacting particles these levels are degenerate. However, the pairing interaction removes this degeneration and pushes down energies of the levels. The maximum shift corresponds to the state $J^{\pi}=0^{+}$. The use of a short-range pairing forces in local potential form

$$
V\left(\overrightarrow{r_{1}}, \overrightarrow{r_{2}}\right)=-V_{0} \delta\left(\overrightarrow{r_{1}}-\overrightarrow{r_{2}}\right)
$$

leads to the relation between energy shifts of level with the total momentum $J \Delta E_{J}[11,12]$ :

$$
\frac{\Delta E_{J}}{\Delta E_{0}}=(2 j+1)\left(\begin{array}{ccc}
j & j & J \\
1 / 2 & -1 / 2 & 0
\end{array}\right)^{2},
$$

where $\Delta E_{0}$ is the shift of the ground state $J^{\pi}=0^{+}$, determined by the pairing energy [13]:

$$
\Delta E_{0}=\Delta_{n n} .
$$

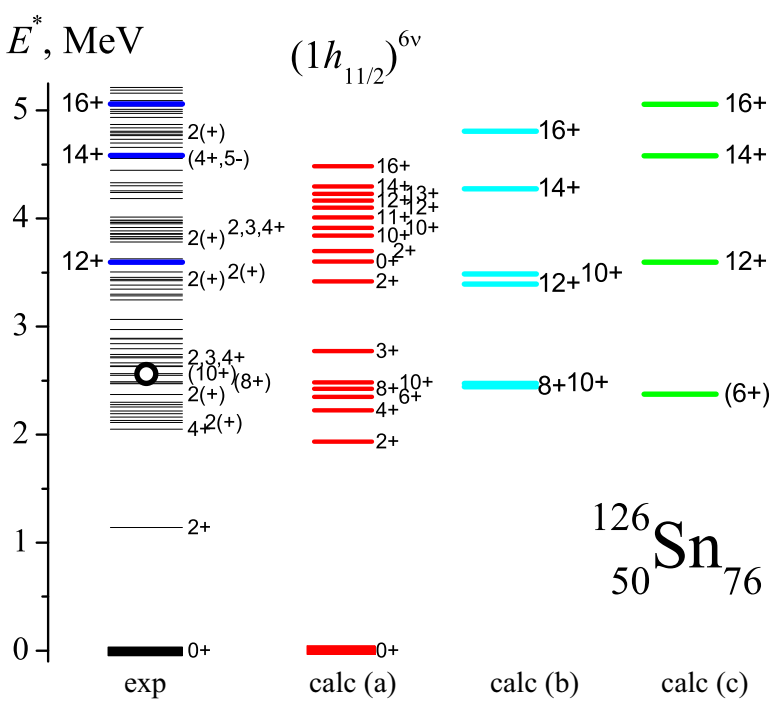

Figure 3. (Color online) The same as in Fig. 2 for the ${ }^{126} \mathrm{Sn}$ isotope.

The neutron pairing energy $\Delta_{n n}$ can be determined as a difference between the two-neutron separation energy in nucleus $(N, Z)$ and twice the neutron separation energy from the nucleus $(N-1, Z)$

$$
\begin{aligned}
\Delta_{n n}(N, Z) & =S_{n n}(N, Z)-2 S_{n}(N-1, Z)= \\
& =S_{n}(N, Z)-S_{n}(N-1, Z) .
\end{aligned}
$$

However, since there is a general decrease in the neutron separation energy, the average value seems to be more realistic

$$
\Delta_{n n}(N, Z)=S_{n}(N, Z)-\frac{S_{n}(N-1, Z)+S_{n}(N+1, Z)}{2}
$$

This expression corresponds to relation between the shift of the state $J^{\pi}=0^{+}$in seniority model and the energy gap $\Delta_{n}$ in the BCS approximation

$$
\Delta_{n n}(N, Z)=2 \Delta_{n},
$$

where the empirical value of $\Delta_{n}$ is determined as an oddeven mass difference parameter [14]. So the position of the ground state multiplet in nuclei with two nucleons (or two holes) over the magic core can be obtained from the experimental mass data.

In the case of several (more than two) nucleons over the magic core the value of seniority $v$ ( $v$ is the number of unpaired nucleons) takes the higher values [15]. The ground state multiplet includes levels with different $v$ up to state with total momentum

$$
J_{\max }=\frac{1}{2} v(2 j-v+1) .
$$

The important consequence of the model is the coincidence of the position of the levels with seniority $v=2$ in the spectra of nuclei with different nucleon numbers. That 
is, in nuclei with four identical nucleons over the closedshell core, the splitting and position of levels of the $v=2$ multiplet can be obtained from Eqs. (2), (4). The structure of $v=4$ multiplet connected with $v=2$ multiplet by means of the coefficients of fractional parentage [16]. It should be noted that the spectra with higher value of seniority is much more complicated than those with $v=2$. For example, in the case of $j=11 / 2$ the $v=4$ spectrum consists of 27 levels with total momentum up to $J^{\pi}=16^{+}$ instead of five levels with $v=2$ with even total angular momentum from $2^{+}$up to $10^{+}$. It is rather difficult to identify the complete structure of the multiplet in the very dense experimental spectra, but some high-spin levels can be identified exactly.

\section{Results}

In view of recent experimental data, especially about the discovery of the isomeric $10^{+}$states in heavy ion collisions [17, 18], the influence of the $j=11 / 2$ level on the structure of yrast levels in nuclei above $N=64$ attracts the permanent attention. The raised interest in studying the role of the $h_{11 / 2}$ subshell in high-seniority excitations is supported by the new experimental data on spinparity assignments of levels in the Sn spectra [8, 10, 19]. In series of experiments at Argonne National Laboratory, where the neutron-rich nuclei produced in collisions of ${ }^{48} \mathrm{Ca}$ and ${ }^{64} \mathrm{Ni}$ projectiles with ${ }^{208} \mathrm{~Pb}$ and ${ }^{238} \mathrm{U}$ targets [8] were studied, excited states above the seniority $v=2$ isomers in even neutron-rich ${ }^{118-128} \mathrm{Sn}$ isotopes were investigated. Shell-model analysis with effective two-body interaction adjusted to the experimental data for ${ }^{130} \mathrm{Sn}$ was carried out with the model space consisting of $1 g_{7 / 2}, 2 d_{5 / 2}$, $3 s_{1 / 2}, 2 d_{3 / 2}$ and $1 h_{11 / 2}$ single-hole neutron states with respect to the ${ }^{132} \mathrm{Sn}$ core. The results of shell-model calculations for ${ }^{124} \mathrm{Sn}$ and ${ }^{126} \mathrm{Sn}$ are shown in Figs. 2 (b), 3 (b), respectively, together with experimental spectra from [6, 7]. Experimental results [8] for levels with spin-parity $J^{\pi}=$ $12^{+}, 14^{+}, 16^{+}$are indicated by blue lines. The shell model calculations based on the model space including $3 s_{1 / 2}$, $2 d_{3 / 2}$ and $1 h_{11 / 2}$ from [10] are shown by green lines in column (c) in both the figures.

Our calculations for the states with seniority $v=2$ and $v=4$ (column (a) in Figs. 2 and 3) were performed for $\delta$-pairing approximation in pure $\left(1 h_{11 / 2}\right)^{n v}$ configuration. We use the estimations of pairing energy (4) based on experimental nuclear mass data [9]: $\Delta_{n n}\left({ }^{124} \mathrm{Sn}\right)=2.63 \mathrm{MeV}$ and $\Delta_{n n}\left({ }^{126} \mathrm{Sn}\right)=2.55 \mathrm{MeV}$. These values are shown in the Figures by open circles. It is seen that these pairing energy values substantially coincide with the energies of $10_{1}^{+}$states. The structure of the multiplet $v=2$ in ${ }^{124} \mathrm{Sn}$ is in good agreement with experimental data. It is difficult to trace the correspondence of the multiplet $v=2$ in ${ }^{126} \mathrm{Sn}$ to the experimental spectrum because of uncertainties in its spin-parity assignments. Nevertheless, it can be stated that $\left(1 h_{11 / 2}\right)^{n v}$ configuration determines the structure of low-energy spectra in ${ }^{124,126} \mathrm{Sn}$ isotopes. The $v=4$ spectra has a lot of levels and not all of them are shown in Figs. 2 and 3, but the position of the high-spin levels is

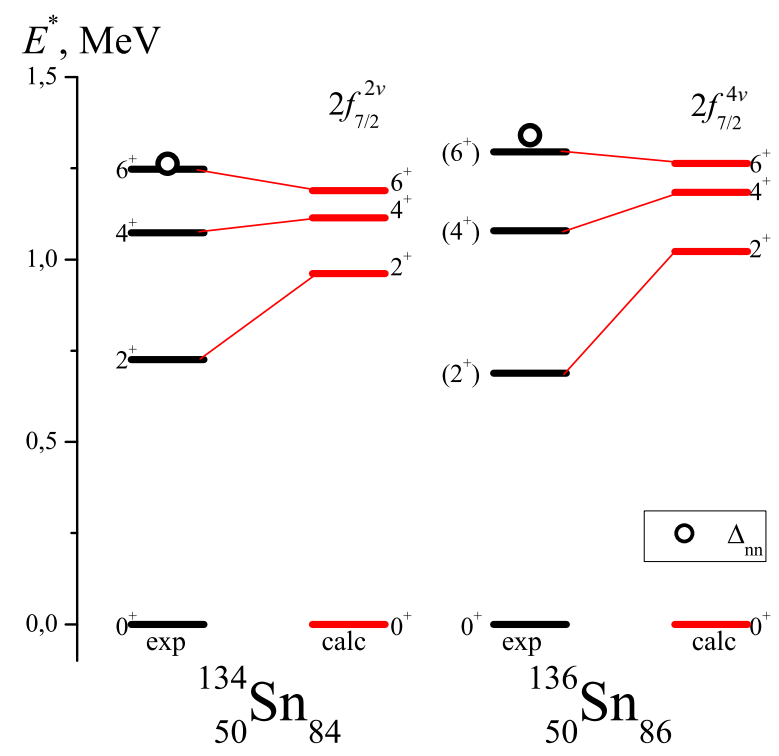

Figure 4. (Color online) Ground state multiplets with $v=2$ in neutron-rich isotopes ${ }^{134,136} \mathrm{Sn}$. Open circles correspond to the neutron pairing energies $\Delta_{n n}$. Experimental data are from $[6,7]$

explicit. The splitting of $v=4$ multiplet less than in shellmodel calculation and this shows the important influence of $3 s_{1 / 2}$ and $2 d_{3 / 2}$ states.

It should be noted that using delta-approximation in $1 h_{11 / 2}$ space gives significantly higher energy values for states with $J^{\pi}=2^{+}$than the experimental one. Comprehensive treatment of low-lying vibrational states in Sn isotopes based on quasiparticle-phonon model shows the one-phonon nature of $2_{1}^{+}$states, and also demonstrate the importance of particle-particle and particle-hole interactions [2, 3]. So, the complex structure of $2_{1}^{+}$doesn't allow to make one-to-one correspondence between this state and $(11 / 2,11 / 2: J=2)$ state in ground state multiplet. But the position of $2^{+}$is very important for the $v=4$ spectra description. In our calculations we use the value from delta-approximation.

The determination of ground state multiplet splitting from the nuclear mass data allows one to make estimations for exotic isotopes of $\mathrm{Sn}$. The double-closed shell isotope ${ }^{132} \mathrm{Sn}$ located near the neutron-drip line. Two next even isotopes ${ }^{134,136} \mathrm{Sn}$ should be considered as one or two neutron pairs on $2 f_{7 / 2}$ subshell over the magic core [20]. Figure 5 represents the experimental spectra of ${ }^{134,136} \mathrm{Sn}$ and the results of calculations for the $v=2$ ground state multiplets. The values of multiplet splitting are determined by $\Delta_{n n}\left({ }^{134} \mathrm{Sn}\right)=1.26 \mathrm{MeV}$ and $\Delta_{n n}\left({ }^{136} \mathrm{Sn}\right)=1.34 \mathrm{MeV}$ and seem to be low in comparison with experiment. This small disagreement may indicate the necessity to consider the admixture the $2 p_{3 / 2}$ state as well as an error of calculating the pair energy near the neutron-drip line.

In Fig. 5, results for light proton-rich $\mathrm{Sn}$ isotopes ${ }^{104,106} \mathrm{Sn}$ are shown. The calculations of the ground state multiplet with seniority $v=2$ were performed for $1 g_{7 / 2}$ state. The values $\Delta_{n n}\left({ }^{104} \mathrm{Sn}\right)=2.70 \mathrm{MeV}$ and $\Delta_{n n}\left({ }^{106} \mathrm{Sn}\right)=2.60 \mathrm{MeV}$ lie too high in comparison with 

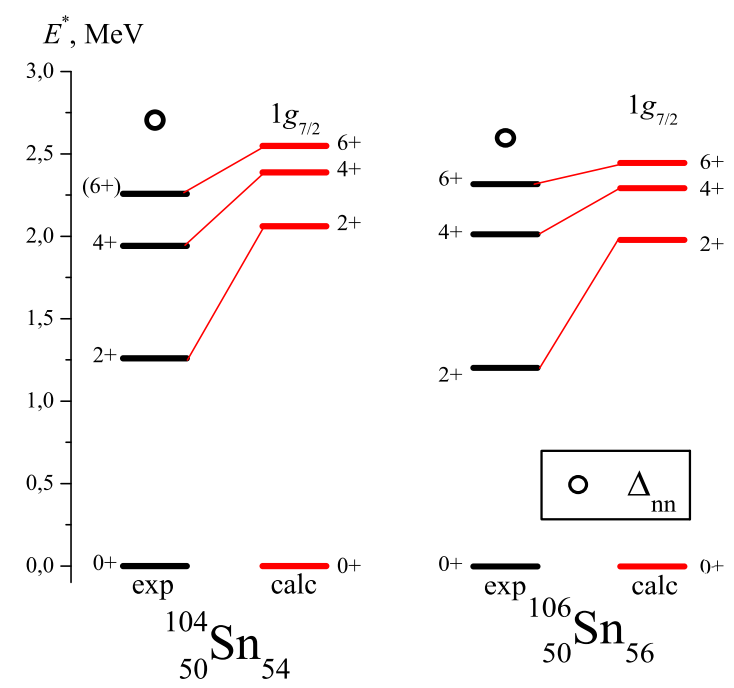

Figure 5. (Color online) Ground state multiplets with $v=2$ in proton-rich isotopes ${ }^{104,106} \mathrm{Sn}$. Open circles correspond to the neutron pairing energies $\Delta_{n n}$. Experimental data are from [6, 7].

level $J^{\pi}=6^{+}$. Near drip lines the mass data are characterized by quite large errors so the even-odd effect estimations based on the three experimental mass points is more preferred [21]. On the other hand, the shell model calculations [22] shows that realistic description of this region should use both the neutron $1 g_{7 / 2}$ and $2 d_{5 / 2}$ subshells and $Z=50$ cross-shell excitations with ${ }^{88} \mathrm{Sr}$ as a closed core.

\section{Conclusions}

The systematics of the ground state multiplets with seniority $v=2,4$ in $\mathrm{Sn}$ isotopes are considered. Thanks to new experimental possibilities a lot of new experimental information appeared on high-seniority levels in ${ }^{120-128} \mathrm{Sn}$ isotopes. Together with a new data on low-energy structure of isotopes near the drip lines this makes the chain of $\mathrm{Sn}$ isotopes an excellent testing ground to probe various theoretical approaches.

The approach based on residual $\delta$-force allows one to estimate the structure of the ground state multiplet using the pair energy $\Delta_{N N}=2 \Delta_{n}$, where the energy gap $\Delta_{n}$ is obtained from the experimental mass data. Strictly speaking, this approach is not applicable to the Sn isotopes since it involves the description of a pure state of pair of nucleons over a double-closed shell nucleus. But the examples of the even isotopes ${ }^{124-128} \mathrm{Sn}$ show that the structure and splitting of $v=2$ multiplet are in good agreement with ex- perimental data. So it can be stated that $\left(1 h_{11 / 2}\right)^{n v}$ configuration defines the position of $J>4$ states of low-energy spectra in ${ }^{124,126} \mathrm{Sn}$ isotopes and some predictions on highseniority $v=4$ multiplet structure can be done.

The double-closed shell isotopes ${ }^{100} \mathrm{Sn}$ and ${ }^{132} \mathrm{Sn}$ are unstable and located near the proton and neutron driplines, respectively. So the neighboring exotic nuclei both the proton-rich isotopes ${ }^{104,106} \mathrm{Sn}$ and the neutron-rich ${ }^{134,136} \mathrm{Sn}$ can be described as a few neutron pairs over the magic core. Our calculations show a good qualitative agreement with the experimental data, but further improvement of the pairing energy estimation is necessary for these regions of exotic isotopes.

\section{References}

[1] A. Bohr, B. Mottelson, Nuclear Structure, vol. 2 (Benjamin, NY, 1975)

[2] A.P. Severyukhin, V.V. Voronov, Nguyen Van Giai, Phys. Rev. C 77, 024322 (2008)

[3] N. Lo Iudice, Ch. Stoyanov, D. Tarpanov, Phys. Rev. C 84, 044314 (2011)

[4] A. Insola et al., Nucl. Phys. A 550, 34 (1992)

[5] V.I. Isakov, Phys. At. Nucl. 76, 828 (2013)

[6] Database «Centre for Photonuclear Experiments Data SINP MSU», http://cdfe.sinp.msu.ru/

[7] National Nuclear Data Center, Brookhaven, Evaluated Nuclear Structure Data File, http://ie.lbl.gov/ensdf/

[8] L.W. Iskra et al., Phys. Rev. C 89, 044324 (2014)

[9] G. Audi et al., Chinese Phys. C 36(12), 1287 (2012); M. Wang et al., Chinese Phys. C 36(12), 1603 (2012)

[10] A. Astier et al., Phys. Rev. C 85, 054316 (2012)

[11] I. Talmi, Simple Models of Complex Nuclei (Harwood Ac. Publ., Chur, 1993), p. 179.

[12] A. De-Shalit, Phys. Rev. 91, 6 (1953)

[13] L.T. Imasheva, et al., Bull. RAS. Physics. 79, 521 (2015)

[14] P. Moller, J.R. Nix, Nucl. Phys. A 536, 20 (1992)

[15] G. Racah, Phys. Rev. 43, 367 (1943)

[16] B.F. Bayman, A. Lande, Nucl.Phys. 77, 1 (1966)

[17] R. Broda et al., Phys. Rev. Lett. 68, 1671 (1992)

[18] C.T. Zhang et al., Phys. Rev C 62, 057305 (2000)

[19] N. Fotiades et al., Phys. Rev. C 84, 054310 (2011)

[20] B.F. Bayman et al., Phys. Rev. C 90, 044322 (2014)

[21] B.S. Ishkhanov, M.E. Stepanov, T.Yu. Tretyakova, Moscow Univ. Phys. Bull. 69, 1 (2014)

[22] L. Coraggio et al., Phys. Rev. C 91, 041301(R) (2015) 